\title{
The Concept of Building a Model of the National Blood Information System
}

\author{
Saif Q. Muhamed ${ }^{1}$, Mohammed Q. Mohammed ${ }^{2}$, Thaker Nayl ${ }^{3}$, Kateryna Chyrkova ${ }^{4}$ \\ ${ }^{1}$ Department Business information technologies / University of information technology and communication \\ saifkassimm@gmail.com \\ ${ }^{2}$ Department of Management information system / University of information technology and communication \\ mqmhf82@gmail.com \\ ${ }^{3}$ Department of Management information system / University of information technology and communication \\ Thaker.nayl@gmail.com \\ ${ }^{4}$ Department Information control system / National University of Radio Electronics / Ukraine, Kharkov \\ yes.chirkova@gmail.com
}

\begin{abstract}
The development of modern information technologies in medicine makes actually the creation of the national Information Systems (IS) for joint activities of medical institutions, improve the quality of health services and improve management in the health sector. One of the components of healthcare is the system of Blood Service (BS). In this work the concept of building the national system is considered on example of the IS of BS. The national IS of BS aims to track relevant information on indicators of the quality of blood products through information integration BS establishments, makes it possible to increase the level of infectious safety and quality of transfusion care. The models of integration IS of BS are offered on the conceptual level in this work for information exchange organization between BS establishments. The analysis of structures of models of integrated systems is carried out to select the rational national IS of BS.
\end{abstract}

\section{Keywords: blood service, integration, information support, data exchange.}

\section{1- INTRODUCTION}

The current state of development of Information Systems (IS), telecommunications networks and technologies, systems and mechanisms to protect information and prospects of their further development allow countries to decide the issues of information space creation of the main fields of activity such as education, science, healthcare, finance, transport and other areas. One of the priority areas of informatization in countries around the world is the healthcare. Currently a great attention is paid to informatization of Blood Services (BS) in context of healthcare development. BS is considered as a structure that combines medical establishments (or their structural units) through the country whose main activity is the collection, processing, testing, storage, distribution, quality assurance of blood products and haemovigilance [1].

World experience studies [2] lead to the conclusion that BS of the country is usually based on regional and territorial principle and includes blood centers, mobile collection units, and hospital blood banks. BS establishments implementing IS to provide information support of business processes. The main problem of $\mathrm{BS}$ is the lack of information exchange mechanism between the BS establishments. However, not all IS of BS have modernization potential to information exchange between other IS. At present, information about donors and persons who are not allowed to perform donor functions located in different databases of BS establishments and related organizations. This procedure of working with data leads to reduce the possibility to track of timely reception of actual data about infectious safety and risks of transfusion reactions and complications of transfusion blood products. The problem solution of designing and implementing the national IS of BS will provide information support of all stages of the BS business process, rapid exchange of relevant data between BS establishments about donors and persons who are not allowed to perform donor functions, statistical and analytical reporting on the country level.

To create a national IS of BS, first need is to analyze the functionality opportunities of IS in BS establishments and define potential opportunities of information interaction with other systems. In turn, this analysis needs to solve a number of questions [3], such as development of the specification system requirements, risk assessment, expert assessment of effectiveness of existing IS of BS, construction of the methodology of evaluation, evaluation of the state of BS technical support, validation of BS, etc. In addition, the problem of organizing the interaction with other IS requires determining the legal principles and organizational solutions to ensure interoperability of IS of BS.

The results of the analysis of the potential opportunities of IS for its information interaction with other systems provide a basis for studying the variants of building a model of the national IS of BS, which must be implementing information interaction between BS establishments, determining formats of data exchange between systems, data structures, etc. 


\section{2- RELATED WORKS}

Distributed and centralized organizations of data storage are the basic approaches of building such class of system as complex integrated systems, which include the national IS of BS. Distributed organization of data storage is typical for Sweden IS of BS, where data transfer between IS of BS establishments is carried out directly through the Internet using the Public Key Infrastructure (PKI technology) of public keys with certificates of encryption and authentication [4]. An example of centralized organization of data storage is the Bulgaria IS of BS, where access to the central database is performed by using the specialized interface through the Internet [5].

The problem of IS integration has received considerable attention in scientific and technical literature [6-8], which addresses various methods based on the use of common standards for unification information exchange, tools and mechanisms of information interaction. The known methods of integration [9] are exchange of files that contain general data, creating a common data base that stores common information, remote procedure call within the systems of information exchange for performing actions or data exchange, asynchronous message exchange, etc. The most common technologies of remote procedure call are Component Object Model (COM), Common Object Request Broker Architecture (CORBA), web-services [10-12].

Solving the problem of IS integration also assumes definition of the universal formats of data transfer. The standards of data exchange and information integration are developed and widely used in healthcare IS, such as HL7, DICOM, ASTM, ASC X12, IEEE/MEDIX, NCPDP [13-15].

The considered methods of integration and approaches to unification based on standards of data exchange and information integration are laid in basis of building national IS of BS.

\section{3- ANALYZING THE MODELS OF BUILDING THE NATIONAL IS OF BS}

Conceptual models of complex integrated IS with centralized, distributed and combined data storage were considered in work, the analysis of features of information interaction between BS establishments in the functioning of the national IS of $\mathrm{BS}$ on the considered approaches to building systems was carried out.

Considering the approach of centralized storage of data for building the national IS of BS model, it is assumed that the currently available information, which own and manage all BS establishments, will be transferred to a single server with a defined access rights. Further management of this information will be through user's workstations in BS establishments, which installed software to interface with the server through the Internet. The scheme of information exchange between BS establishments with central data storage is shown in figure 1.

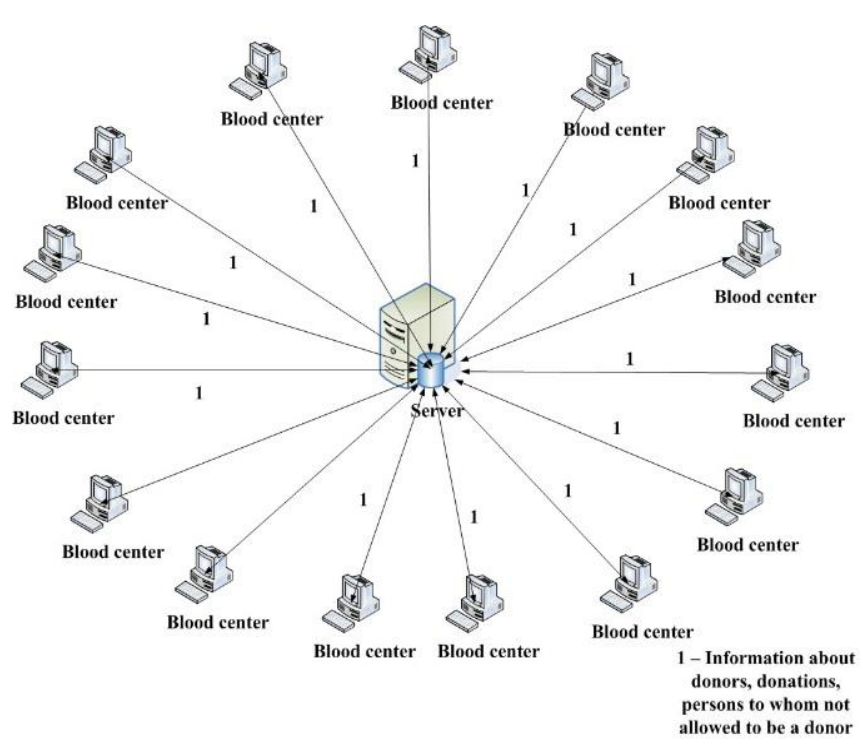

Figure 1: The scheme of information exchange between BS establishments with central data storage

The main advantages of organizing national IS of BS shown on the scheme in figure 1 are:

- ease of centralized management and administration at the country level;

- lack of data duplication;

- ease of access control and modernization;

- existence of single documentation for system implementation and operation;

- ease of consolidated statistical and analytical information on the country level;

- Integrity and consistency of data on the country level.

The main disadvantages of the concept of building national IS of BS according to the scheme with centralized data storage are:

- need for continued access to the central server;

- need to support different configurations of technical equipment that already has operated in BS establishments;

- complexity of the system;

- Need to ensure continuous secure communication channel to the server.

Building the model of national IS of BS with distributed data storage assumes that each BS establishment has IS based on "their own" server to which if necessary will interact other BS establishment IS. The scheme of information exchange 
between $\mathrm{BS}$ establishments with distributed data storage is shown in figure 2.

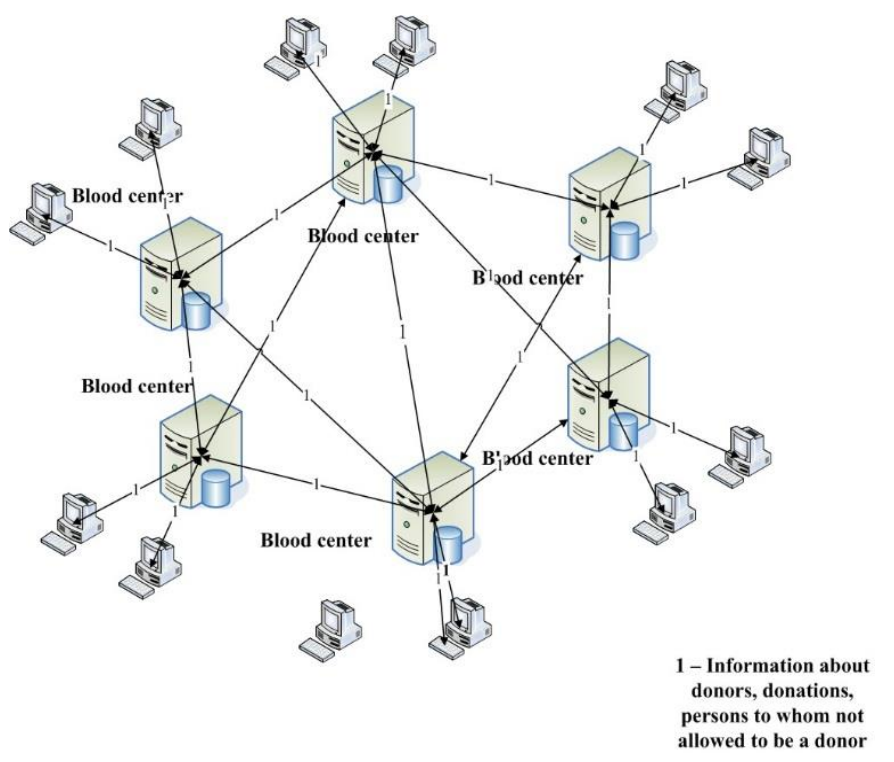

Figure 2: The scheme of information exchange between BS establishments with distributed data storage

The main advantages of organizing the national IS of BS that shown on the scheme in figure 2 are:

- ease of system;

- possibility of autonomous data operation between BS establishments;

- Short time to implementation of new functionality in the system.

The main disadvantages of building the national IS of BS with distributed data storage are:

- significant costs on IS technical equipment in BS establishments;

- significant costs for development of BS establishments IS;

- complex actual development and correct documentation for system;

- need to develop and maintain module of data synchronization;

- data duplication;

- closeness of BS establishments IS;

- lack of documentation description of data sets, that are transferred between systems;

- high complexity of organizing information exchange between systems.

In addition, traditional approaches with centralized and distributed data storage can also consider a combined approach taking into consideration features of internal information communication between BS establishments for building the national IS of BS. The figure 3 is showing the scheme of information exchange between BS establishments with a combined approach. In this approach, for building the national IS of BS, it is expedient to create the system with centralized data storage at the regional level. The integration of regional systems at the country level will allow using web-services. Web-services will provide data exchange between IS of BS establishments through the Internet regardless of programming language and interface. The integration of IS in the national IS of BS using a combined approach requires unification of data exchange formats in the IS.

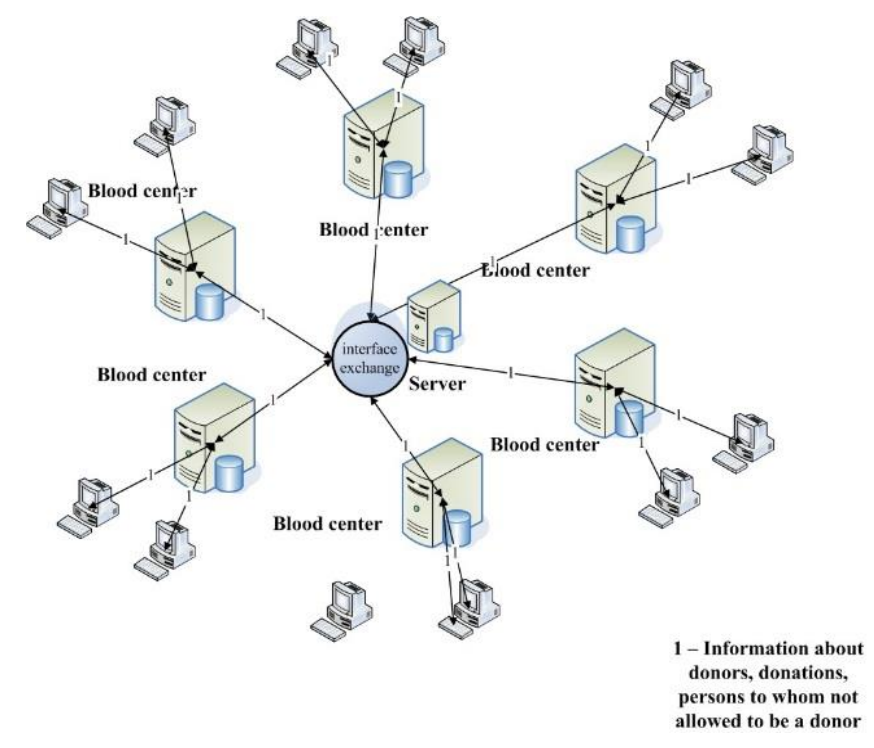

Figure3: The scheme of information exchange between BS establishments with combined data storage

The main advantages of building the national IS of BS with combined approach are:

- rapid deployment of the national IS of BS based on IS that already has operated in BS establishments by developing one interface exchange;

- lack of necessity to solve the problem of selection and implementation of the single IS in BS establishments at the regional level;

- independency of programming languages and technologies used in BS establishments IS;

- possibility of the iterative implementation of functional of the national IS of BS;

- described structure of data sets transferred in the national IS of BS.

The main disadvantages of concept of building the national IS of BS that is shown on the scheme in figure 3 are:

- need for a central server;

- cost of acquisition and maintenance of the central server; 
- complexity of developing a web service.

Selecting the variant of realization for the design of building the national IS of BS can be performed by the generalized criterion. The generalized criterion is proposed to form based on partial indicators that are defined in the analysis of advantages and disadvantages of decisions based on the models of building the national IS of BS. The generalized criterion is as follows:

$K r=\Phi\left(t_{q}, t_{u p}, o p r\right) \rightarrow \min$,

$C \leq C_{\text {const }}$,

$R \leq R_{\text {const }}$,

$t \leq t_{\max }$

Where, $t_{q}$ is the time of implementating the request to the national IS of BS;

$t_{u p}$ is the time of saving and updating data in the national IS of BS;

opr is the number of operations performed by using the national IS of BS;

$C \leq C_{\text {const }}$ is the cost of designing solution to create the national IS of BS within the budget;

$R \leq R_{\text {const }}$ - are the resources needed for building the national IS of BS, subjected to restrictions;

$t \leq t_{\text {max }}$ - is the time of creating the national IS of BS in deadlines;

The characteristics of the working the national IS of BS can be determined experimentally or by analogy with existing solutions. Indicators of cost, resources and time of realization of project decisions on the creation of national IS of BS is supposed to define and clarify the expert way. An alternative variant of evaluating the solutions of building national IS of BS is using only methods of expert evaluation. These methods can determine the qualitative effects of the implementation and maintenance of national IS of BS, but is not excluding the high cost of attracting highly qualified experts, the difficulty of organizing the expertise because of the lack of universal views on the creation of a common information space, especially in such strategic areas as blood services. Therefore, the proposed evaluation method, based on quantitative indicators provides a certain level of objectivity of judgment when making decisions.

\section{4- CONCLUSION}

Today, great attention is paid to the realization of data exchange between $\mathrm{BS}$ establishments to improve safety infectious of blood products. Providing data integration at the national level is a difficult task. Several approaches of building a national IS of BS that has its advantages and disadvantages were considered. The choice of approach of building the national IS of BS of each country depends on the specification of IS that integrate, interchange data formats, by using price policy, strategic objectives of creating such systems with considering organizational and legal preconditions of constructing country BS and features of the country's legislative framework. An important aspect of building the national IS of BS is the use of international industry standards and practices in automation, such as GAMP. Taking into account the above and based on the proposed criteria, the model of building the national IS of BS for the certain country can be defined.

\section{REFERENCES}

[1] J.R. Cruz, "Basic components of a national blood system", Rev Panam Salud Publica, vol.13 n.2-3, pp. 79-84, 2003.

[2] "National standards for blood transfusion service", Blood Safety Program, Edition 1, 2013.

[3] ISBT Guidelines for Validation of Automated Systems in Blood Establishments", International Journal of Blood Transfusion Medicine, Vox Sanguinis, pp. 1-19, 2010.

[4] "ProSang - the leading blood management system in Scandinavia"[Online]. Available: www.prosang.com. [Accessed: Sept. 21, 2016].

[5] Project BG051PO001-6.2.10, "Development and implementation of Automated Information System / AMS / centers and departments of Haematology in Bulgaria", Sept. 2011. [Online]. Available: http://ncth.bg/projects/305-ais. [Accessed: Sept. 22, 2016].

[6] S. Kitsiou, V. Manthou, and M. Vlachopoulou, "A Framework for the Evaluation of Integration Technology Approaches in Healthcare", Research Gate, 2006.

[7] H. Panetto, J. Cecil, "Information systems for enterprise integration, interoperability and networking: theory and applications Enterprise Information Systems, pp. 1-6, 2013.

[8] D. Litan, A.M. Mocanu, L. Copcea, I. Surugiu, O. Rabuta,"Information Systems Integration, a New Trend in Business", Recent Researches in Communications, Electrical and Computer Engineering, pp. 250-255, 2011.

[9] V. Asproth, "Integrated Information Systems - A Challenge for Long-Term Digital Preservation", Interdisciplinary Journal of Information, Knowledge, and Management, pp. 89-96, 2007.

[10] Q. A. Kester, "Using SOA with Web Services for effective Integration of Hospital Information Systems via an Enterprise Service Bus", International Journal of Research in Engineering and Advanced Technology, pp. 1-5, 2011. 
[11] J.K. Zhang, W. Xu, "Web Service-based Healthcare Information System (WSHIS): A Case Study for System Interoperability Concern in Healthcare Field", In International Conference on Biomedical and Pharma ceutical Engineering, pp. 588 - 594, 2006.

[12] Q. H. Mahmoud, "Service-Oriented Architecture (SOA) and Web Services: The Road to Enterprise Application Integration (EAI)", April, 2006.[Online]. Available: http://www.oracle.com/technetwork/systems/soa142870.html. [Accessed: Sept. 22, 2016].

[13] N.Sabooniha, D. Toohey, K.Lee "An Evaluation of Hospital Information Systems Integration Approaches", In: International Workshop on Recent Advances in Medical Informatics (RAMI-2012), 3 5 August, Chennai, India

[14] E. Monteiro, "Integrating Health Information Systems: A Critical Appraisal", Methods Inf Med 4, pp. 428-432, 2003.

"Health Level 7" [Online]. Available:

http://www.hl7.org. [Accessed: Sept. 21, 2016] 